\title{
Avaliação do Teor de \\ Nitrito em Apresuntados \\ Armazenados em \\ Diferentes Temperaturas
}

\author{
Gustavo H. F. Faria, Aretha F. Moreno, Miriam F. A. Silveira, Robson \\ M. Geraldine \& Deivis M. Carvalho
}

O estudo objetivou avaliar o teor de nitrito, o $\mathrm{pH}$ e a cor de apresuntados, armazenados a $4 \pm 1^{\circ} \mathrm{C}$ e a $8 \pm 1^{\circ} \mathrm{C}$, durante 40 dias. A temperatura de armazenamento afetou significativamente o teor de nitrito, visto que as amostras a $8 \pm 1^{\circ} \mathrm{C}$ apresentaram menor teor, em todos os tempos avaliados. $\mathrm{O}$ tempo de armazenamento exerceu efeito significativo sobre o teor de nitrito das amostras, em ambos os tratamentos. $\mathrm{O}$ pH médio das amostras foi de 6,6. A cor dos apresuntados não foi alterada significativamente pelas temperaturas utilizadas, durante o tempo de armazenamento. A diferença de cor somente foi observada no $40^{\circ}$ dia de armazenamento, em ambas as temperaturas.

Palavras - chave: carne; processamento; nitrito.

The study aimed to evaluate the nitrite content, $\mathrm{pH}$ and pressed ham color stored at 4 $\pm 1^{\circ} \mathrm{C}$ and $8 \pm 1^{\circ} \mathrm{C}$, for 40 days. The storage temperature significantly affected nitrite content, where as the samples at $8 \pm 1^{\circ} \mathrm{C}$ had lower contents in all time periods studied. The storage time had a significant effect on the nitrite content of the samples in both treatments. The average $\mathrm{pH}$ of the samples was 6.6. There was no significant change in color, during 30 days of storage of pressed ham, used in temperatures. The color of the pressed ham was not significantly affected by the temperatures used during the storage time. A color difference was observed only after 40 day of storage at both temperatures .

Keywords: meat; processing; nitrite. 


\section{Introdução}

A carne é uma matéria-prima altamente perecível, portanto, as condições de armazenamento são determinantes na sua conservação. Diante dessa característica, a fabricação de produtos curados não só aumenta a diversidade de produtos oferecidos ao consumidor, mas também é uma forma de propiciar o aumento da validade das carnes ${ }^{1}$.

O uso de aditivos na industrialização desses produtos é regulamentado e deve ser controlado para que seja seguido seu padrão característico e garantida a segurança do consumidor. Os sais de cura, nitratos e nitritos de sódio e de potássio exercem papel importante tanto na conservação de produtos cárneos, inibindo o crescimento de micro-organismos patogênicos, como o Clostridium botulinum, quanto na manutenção de características sensoriais determinantes na sua atratividade. Estes sais são adicionados em produtos industrializados, como presunto, apresuntado, salsicha, mortadela, entre outros, no processo de cura, proporcionando a fixação da cor, sabor, aroma e melhoria no rendimento ${ }^{2}$.

Existe grande preocupação da comunidade científica com possíveis efeitos tóxicos que esses sais podem causar à saúde humana ${ }^{3,4}$. $\mathrm{O}$ nitrito pode se combinar com a hemoglobina, transformando-a em metahemoglobina, reduzindo a eficiência no transporte de oxigênio, principalmente, em crianças, podendo causar sintomas como cianose, fadiga, dispneia, cefaleia e até morte ${ }^{5}$. Outro possível efeito é a reação entre o nitrito e as aminas secundárias e terciárias, formando $\mathrm{N}$-nitrosaminas, que podem apresentar atividade carcinogênica, mutagênica e teratogênica ${ }^{6}$. A toxicidade do consumo de alimentos que contenham nitritos e nitratos está relacionada à quantidade ingerida, por isso se faz importante o controle da quantidade adicionada no produto.

Em presença de nitrito e outros subprodutos da reação, os pigmentos da carne podem sofrer várias alterações que dependem de fatores intrínsecos e extrínsecos. A reação inicial consiste na conversão da mioglobina e oximioglobina em metamioglobina. Esta reage com óxido nítrico formando o complexo nitrosometamioglobina, que pode reduzir-se ao nitrosomioglobina, pigmento da carne curada sem ação do calor. O nitrosohemocromo é o pigmento final que deve estar presente nas carnes curadas submetidas ao aquecimento. Apresenta coloração rósea característica de produtos curados?
A dosagem de nitrato/nitrito empregada durante o processamento dos produtos cárneos é um fator de grande importância. Não apresenta relação direta clara com os valores residuais, visto que está relacionado ao grau de complexidade das reações e da matriz cárnea e às interações entre ingredientes. Diante do exposto, o presente estudo teve como objetivo avaliar o efeito de diferentes temperaturas de armazenamento de apresuntados quanto ao teor de nitrito, $\mathrm{pH}$ e cor, durante 40 dias.

\section{Metodologia}

Os apresuntados foram processados com carne suína (paleta) e toucinho, resfriados a $4^{\circ} \mathrm{C}$, adquiridos no Mercado Municipal da cidade de Goiânia-Goiás. O sal de cura (nitrito de sódio), o estabilizante (tripolipolifosfato de sódio), o antioxidante (ascorbato de potássio), os condimentos e a fécula de mandioca foram fornecidos pela empresa Fego Alimentos LTDA e utilizados conforme recomendação descrita no rótulo. Todo procedimento foi realizado seguindo rigorosamente as Boas Práticas de Fabricação ${ }^{8}$.

A primeira etapa do processamento consistiu na limpeza da carne suína e retirada do excesso de gordura. Em seguida, a carne e o toucinho foram moídos em disco de 5 $\mathrm{mm}$. Os ingredientes açúcar, sal, estabilizante, condimentos e sal de cura foram adicionados à massa e misturados, manualmente, por 5 minutos. Em seguida, o antioxidante foi acrescentado e misturado até obter massa uniforme, que foi acondicionada em sacos plásticos de polietileno e armazenada sob refrigeração $\left(4 \pm 2{ }^{\circ} \mathrm{C}\right.$ ), por 24 horas, para ocorrer a cura. Após o processo de cura, a fécula de mandioca foi adicionada à massa e misturada por 5 minutos. A massa foi colocada em formas de aço inoxidável, previamente forradas com filme de celulose regenerada, em quantidade suficiente para obtenção de apresuntados com, aproximadamente, $1,0 \mathrm{Kg}$. Após a prensagem da massa, foi realizado o cozimento em água a $80 \pm 2{ }^{\circ} \mathrm{C}$, durante 80 minutos. Em sequência à cocção, os apresuntados, ainda enformados, foram resfriados em refrigerador rápido com circulação de ar forçado, até temperatura interna de, aproximadamente, $1^{\circ} \mathrm{C}$. Foi feita a desenformagem dos apresuntados e retirado o filme de celulose regenerada. Os apresuntados foram fracionados, de forma a obter amostras de $100 \mathrm{~g}$. As amostras foram embaladas a vácuo em filme aluminizado e armazenadas sob refrigeração, utilizando-se duas temperaturas, $4 \pm 1^{\circ} \mathrm{C}$ e $8 \pm 1^{\circ} \mathrm{C}$. 
Analisaram-se as amostras quanto ao teor de nitrito, pH e cor, a cada 10 dias de armazenamento, iniciandose no tempo T0 (data de fabricação) e finalizando-se no tempo T40 (40 dias de armazenamento).

$\mathrm{O}$ teor de nitrito e o $\mathrm{pH}$ foram determinados, conforme metodologias descritas nas NORMAS ANALÍTICAS DO INSTITUTO ADOLFO LUTZ ${ }^{9}$. Para a determinação do teor de nitrito, baseou-se nas reações de diazotação de nitritos com ácido sulfanílico e copulação com cloridrato de alfa-naftilamina em meio ácido, formando o ácido alfanaftilamino-p-azobenzeno-p-sulfônico de coloração rósea. $\mathrm{O}$ produto resultante foi determinado espectrofotometricamente a $540 \mathrm{~nm}$.

A cor dos apresuntados foi medida em colorímetro de bancada (Hunter Associates Laboratory Inc - Coloquest II, Reston, EUA), operando no sistema CIE (L*, $\left.a^{*}, b^{*}\right)$. Os valores de $\mathrm{L}^{*}$ (Luminosidade), $\mathrm{a}^{*}$ (intensidade da cor verde a vermelho) e $b^{*}$ (intensidade da cor amarela a azul) foram obtidos após a calibração do equipamento (padrão branco e preto), utilizando o software Universal v 3.6 10. Com os dados obtidos foi calculada a diferença de cor $\Delta \mathrm{E}^{*}$ (Equação 1).

$$
\Delta \mathrm{E}=\left(\Delta \mathrm{L}^{2}+\Delta \mathrm{a}^{2}+\Delta \mathrm{b}^{2}\right)^{1 / 2}
$$

Em que: $\Delta \mathrm{L}, \Delta \mathrm{a}, \Delta \mathrm{b}$ são os diferenciais entre os parâmetros de cor das amostras.

$\mathrm{O}$ experimento foi conduzido em delineamento em blocos com parcelas subdivididas no tempo, com 3 repetições, sendo os resultados submetidos a análise de variância (ANOVA) e a comparação entre as médias pelo teste de Tukey $(\mathrm{p} \leq 0,05)$, utilizando o programa Statistica 7.0.

\section{Resultados e Discussão}

O processamento dos apresuntados foi considerado satisfatório, visto que, ao final do processo de fabricação, foi possível identificar aspectos característicos do produto, como aroma, coloração rósea, confirmando a formação do pigmento nitrosohemocromo após o cozimento do produto, e textura coesa. Essas características apresentaram-se semelhantes às dos produtos encontrados no comércio.

Observou-se que as amostras apresentaram teor médio de nitrito de 140,48 $\pm 0,77 \mathrm{mg} \cdot \mathrm{kg}-1$ após a fabricação (T0), estando, portanto, dentro do limite máximo permitido pela legislação brasileira em vigor, que é de 150 mg.kg- $1^{11}$.

A temperatura de armazenamento afetou significativamente $(\mathrm{p} \leq 0,05)$ o teor de nitrito das amostras de apresuntado nos tempos avaliados (Tabela 1). As amostras armazenadas a $8 \pm 1{ }^{\circ} \mathrm{C}$ apresentaram menor teor de nitrito em relação às amostras submetidas ao tratamento a $4 \pm 1{ }^{\circ} \mathrm{C}$, sugerindo que temperaturas de armazenamento mais altas favorecem a degradação do nitrito. Esse aditivo está sujeito a uma série de reações relacionada às suas propriedades como alimento. A quantidade adicionada no momento do processamento será de certa forma consumida, e ao longo do tempo será identificada uma redução na quantidade de nitrito residual detectável, que pode ser mais ou menos rápida, dependendo de fatores intrínsecos, inerentes à matériaprima, e/ou extrínsecos, inerentes às condições de armazenamento $^{12}$, como a temperatura. A concentração de nitrito em carnes cai sensivelmente durante o processo de aquecimento e armazenamento, sendo que esta diminuição ocorre mais em altas temperaturas do que em baixas temperaturas de armazenamento ${ }^{13}$.

Tabela 1. Teor de nitrito em apresuntados armazenados a $4 \pm 1{ }^{\circ} \mathrm{C}$ e a $8 \pm 1^{\circ} \mathrm{C}$ por 40 dias.

\begin{tabular}{|c|c|c|c|c|c|}
\hline \multirow{5}{*}{ Temperatura $\left({ }^{\circ} \mathrm{C}\right)$} & \multicolumn{5}{|c|}{ Teor de Nitrito $\left(\mathrm{mg} . \mathrm{Kg}^{-1}\right)$} \\
\cline { 2 - 6 } & $\mathrm{T} 0$ & $\mathrm{~T} 10$ & $\mathrm{~T}$ Tempo de armazenamento (dias) & \multicolumn{2}{c|}{$\mathrm{T} 30$} \\
\hline 4 & $140,45 \pm 0,70 \mathrm{Aa}$ & $128,71 \pm 0,56 \mathrm{Ba}$ & $102,46 \pm 0,30 \mathrm{Ca}$ & $88,06 \pm 0,68 \mathrm{Da}$ & $19,65 \pm 0,04 \mathrm{Ea}$ \\
\hline 8 & $140,52 \pm 0,84 \mathrm{Aa}$ & $119,40 \pm 0,32 \mathrm{Bb}$ & $91,39 \pm 1,01 \mathrm{Cb}$ & $67,47 \pm 0,54 \mathrm{Db}$ & $11,74 \pm 0,07 \mathrm{~Eb}$ \\
\hline
\end{tabular}

Médias seguidas das mesmas letras minúsculas (dentro de cada tempo) ou maiúsculas (dentro de cada temperatura) não apresentam diferença significativa entre si, pelo teste de Tukey $(\mathrm{p}>0,05)$. 
Verificou-se que o tempo de armazenamento exerceu efeito significativo $(\mathrm{p}<0,05)$ sobre o teor de nitrito das amostras, em ambas as temperaturas. A redução do teor de nitrito é explicada por CASSENS et al. ${ }^{14}$, que consideram o tempo e a temperatura de armazenamento como fatores que podem contribuir para a diminuição do nitrito residual detectável, à medida em que ele se combina com o pigmento da carne ou em outras reações. Segundo Ferraccioli1 ${ }^{12}$, o nível de nitrito residual detectado em carnes é bastante reduzido em relação ao teor adicionado, pois esse pode reagir com aminas da carne durante o processamento e armazenamento.

Observou-se que as amostras apresentaram $\mathrm{pH}$ médio de 6,6. Não houve diferença significativa $(p>0,05)$ entre tratamentos, nos tempos avaliados. Também Silva et al. ${ }^{15}$, analisando valores de $\mathrm{pH}$ em patês de presunto, em função do tempo de armazenamento, não encontraram diferenças significativas. Das cinco amostras de patê analisadas, quatro apresentaram $\mathrm{pH}$ próximo de 6,5.

Observa-se, pelos resultados apresentados na Tabela 2 , que não houve alteração significativa $(\mathrm{p}>0,05)$ da cor característica de produtos curados durante o armazenamento dos apresuntados, nas temperaturas utilizadas. Diferenças de cor foram observadas apenas entre o $30^{\circ}$ e o $40^{\circ}$ dia de armazenamento, para ambas as temperaturas. Os sais de cura influenciam fortemente na estabilidade da cor dos produtos ${ }^{16}$.

A cor rósea, característica dos produtos curados cozidos, como é o caso dos apresuntados produzidos nesse trabalho, é formada por meio de modificações químicas entre os pigmentos naturais da carne e suas reações com o nitrito. Nessas reações, ocorre formação de nitrosomioglobina de cor vermelho-rosado que, com o cozimento do produto, é transformado em nitrosohemocromo, pigmento responsável pela coloração desses produtos ${ }^{17}$.
A embalagem a vácuo e opaca (filme aluminizado), utilizada no acondicionamento dos apresuntados, contribuiu para a manutenção da estabilidade da cor rósea, durante o período avaliado, visto que o pigmento nitrosohemocromo é instável ao oxigênio e à luz ${ }^{18}$. A cor e sua estabilidade são parâmetros importantes na avaliação da qualidade global da carne e de seus derivados ${ }^{19}$. São atributos determinantes para decisão de compra pelos consumidores, a cor e a aparência dos produtos cárneos, influenciando significativamente na percepção de seu frescor $^{20}$.

\section{Conclusão}

A temperatura e o tempo de armazenamento dos apresuntados influenciaram o teor de nitrito, porém não afetaram o $\mathrm{pH}$ do produto. Não houve alteração significativa da cor característica de produtos curados durante o armazenamento dos apresuntados, nas temperaturas utilizadas. A diferença de cor somente foi observada no $40^{\circ}$ dia de armazenamento, em ambas as temperaturas.

\section{Referências}

1. Duarte, M.T. Avaliação do teor de nitrito de sódio em linguiças do tipo frescal e cozidas comercializadas no estado do Rio de Janeiro. 2010. Tese (Doutorado em medicina veterinária). Universidade Federal Fluminense, Niterói, RJ.

2. Araújo, J. M. A. Química de alimentos: teoria e prática. $5^{\mathrm{a}}$ ed. UFV: Viçosa, 2011.

3. Ferguson, L. R. Meat Sci.. 2010, 84, 308.

4. Araújo, P. F.; Rodrigues, R. S. Revista Higiene Alimentar. 2008, $22,54$.

5. Okafor, P. N.; Ogbonna, V. I. J. Food Compos. Anal.. 2003, 16, 213.

Tabela 2. Diferença de cor em apresuntados armazenados a $4 \pm 1^{\circ} \mathrm{C}$ e a $8 \pm 1^{\circ} \mathrm{C}$ por 40 dias.

\begin{tabular}{|c|c|c|c|c|c|}
\hline \multirow{5}{*}{ Temperatura $\left({ }^{\circ} \mathrm{C}\right)$} & \multicolumn{5}{|c|}{ Diferença de cor $(\Delta \mathrm{E})$} \\
\cline { 2 - 6 } & $\mathrm{T} 0$ & $\mathrm{~T} 10$ & $\mathrm{~T}$ Tempo de armazenamento (dias) & $\mathrm{T} 20$ & $\mathrm{~T} 30$ \\
\hline 4 & $33,56 \pm 0,45 \mathrm{Aa}$ & $33,99 \pm 0,78 \mathrm{Aa}$ & $34,16 \pm 0,44 \mathrm{Aa}$ & $34,55 \pm 0,55 \mathrm{Aa}$ & $35,96 \pm 0,28 \mathrm{Ba}$ \\
\hline 8 & $33,70 \pm 0,81 \mathrm{Aa}$ & $33,70 \pm 0,63 \mathrm{Aa}$ & $34,83 \pm 0,53 \mathrm{Aa}$ & $35,14 \pm 0,73 \mathrm{Aa}$ & $35,60 \pm 0,47 \mathrm{Ba}$ \\
\hline
\end{tabular}

Médias seguidas das mesmas letras minúsculas (dentro de cada tempo) ou maiúsculas (dentro de cada temperatura) não apresentam diferença significativa entre si, pelo teste de Tukey $(\mathrm{p}>0,05)$. 
6. Martins, D. I.; Mídio, A. F.Toxicologia de alimentos. $2^{\mathrm{a}}$ ed. Varela: São Paulo, 2000.

7. Roça, R. O. Tecnologia da carne e produtos derivados. Faculdade de Ciências Agronômicas UNESP: Botucatu, 2010.

8. Brasil. Agência Nacional de Vigilância Sanitária. Resolução RDC $\mathrm{n}^{\circ} .216,2004$.

9. Instituto Adolfo Lutz. Normas Analíticas do Instituto Adolfo Lutz.

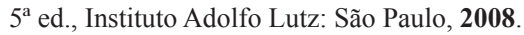

10. Hunterlab. User's manual hunter associates laboratory, Reston, 1997.

11. Brasil. Ministério da Agricultura, Pecuária e Abastecimento. Instrução normativa $\mathrm{n}^{\circ} 51$, de 29 de dezembro de 2006.

12. Ferraccioli, V. R. Avaliação da qualidade de salsichas do tipo hot dog durante o armazenamento. 2012. Dissertação (Mestrado em engenharia de processos químicos e bioquímicos). Centro Universitário do Instituto Mauá de Tecnologia, São Caetano do Sul, SP.

13. Jay, J. M.; Tondo, E. C. Microbiologia de alimentos. $6^{\mathrm{a}}$ ed. Artmed: Porto Alegre, 2005.

14. Cassens, R.; Greaser, M.; Ito, T.; Lee. M. Food Technol.. 1979, $33,46$.

15. Silva, J. G. et al. Ciênc. Tecnol. Aliment.. 2003, 23, 10.

16. Pardi, M.C.; Santos, I.F.; Souza, E.R.; Pardi, H. S. Ciência, higiene e tecnologia da carne. Ed. UFG: Goiânia, 2007.

17. Ordóñez, J. A. P. et al. Tecnologia de alimentos. $2^{\mathrm{a}}$ ed. Artmed: Porto Alegre, 2005.

18. Faria, J. A. F.; Felício, P. E.; Neves, M. A.; Romano, M. A. Revista Tec Carnes. 2001, 3, 16.

19. Troy, D. J.; Kerry, J. P. Meat Sci.. 2010, 86, 214.

20. Ramos, E. M.; Gomide, L. A. M. Avaliação da qualidade de carnes: fundamentos e metodologias. Ed. UFV: Viçosa, 2007.

\section{Gustavo H. F. Faria; Aretha F. Moreno; Miriam F. A. Silveira*; Robson M. Geraldine \& Deivis $M$. Carvalho}

Endereço dos autores: Universidade Federal de Goiás, Escola de Agronomia, Campus Samambaia, Rodovia Goiânia/Nova Veneza, Km 0, Goiânia, Goiás, Brasil - CEP 74690-900.

*E-mail: miriamfas.ufg@gmail.com; 